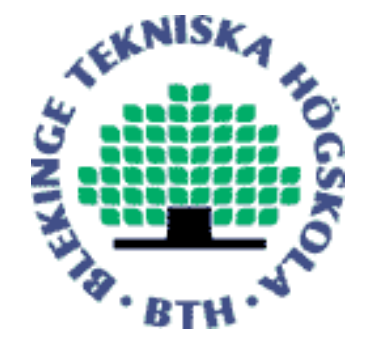

Copyright (C) 2010 IEEE.

Citation for the published paper:

APPrOVE: Application-oriented Validation and Evaluation of Supervised Learners

Niklas Lavesson, Paul Davidsson

IEEE Intelligent Systems

2010 London

This material is posted here with permission of the IEEE. Such permission of the IEEE does not in any way imply IEEE endorsement of any of BTH's products or services Internal or personal use of this material is permitted. However, permission to reprint/republish this material for advertising or promotional purposes or for creating new collective works for resale or redistribution must be obtained from the IEEE by sending a blank email message to pubs-permissions@iee.org.

By choosing to view this document, you agree to all provisions of the copyright laws protecting it. 


\section{APPrOVE: Application-oriented Validation and Evaluation of Supervised Learners}

\author{
Niklas Lavesson \\ School of Computing \\ Blekinge Institute of Technology \\ SE-372 25 Ronneby, Sweden \\ Email: Niklas.Lavesson@bth.se
}

\author{
Paul Davidsson \\ School of Computing \\ Blekinge Institute of Technology \\ SE-372 25 Ronneby, Sweden \\ Email: Paul.Davidsson@bth.se
}

\begin{abstract}
Learning algorithm evaluation is usually focused on classification performance. However, the characteristics and requirements of real-world applications vary greatly. Thus, for a particular application, some evaluation criteria are more important than others. In fact, multiple criteria need to be considered to capture application-specific trade-offs. Many multicriteria methods can be used for the actual evaluation but the problems of selecting appropriate criteria and metrics as well as capturing the trade-offs still persist. This paper presents a framework for application-oriented validation and evaluation (APPrOVE). The framework includes four sequential steps that together address the aforementioned problems and its use in practice is demonstrated through a case study.
\end{abstract}

Index Terms-classification, evaluation, supervised learning

\section{INTRODUCTION}

Consider supervised classification learning, where the task is to infer general descriptions of concepts, i.e., classifiers, given examples with known class labels. The dominating quality criterion for learning algorithms and classifiers on this task is accuracy, which is often evaluated using metrics such as success rate or the area under the ROC curve (AUC). However, it has been argued that, for a particular application, some evaluation metrics are more appropriate than others [1]. Thus, the choice of metric(s) used to evaluate accuracy should be based on the application at hand. We agree on this assessment and further argue that, for a particular application, some evaluation criteria (e.g.: complexity, comprehensibility, accuracy, and efficiency) are more important than others. The question is how to identify which criteria best represent the quality of the candidate algorithms and classifiers with respect to a certain application.

Supervised classification learning algorithms are used in various applications, e.g., diagnosis, text categorization and image recognition. A particular type of application, e.g., image recognition, and a particular problem to be solved, e.g., the recognition of known criminals from a database of identification card photos, could depend on the fulfillment of a certain set of criteria, e.g.: accuracy, efficiency, and complexity. However, the requirements may not be explicitly defined as a set of criteria or evaluation metrics and the relative importance of the criteria can be difficult to estimate. There is an abundance of learning algorithms to choose from when facing a classification problem. The fundamental question is how to define and evaluate the quality of a learning algorithm as it pertains to the particular problem studied. Although few would argue against the importance of this question, it has in fact received little attention by the research community. The reason behind this contradiction is not clear but a contributing factor may be that of the traditionally strong focus on accuracy. Whether the aim is to present a novel algorithm or to compare algorithms on some specific real-world problem, an overwhelming majority of the published work is solely concerned with accuracy. Hence, this criterion is probably viewed by many as the obvious benchmark for conclusively determining algorithm or classifier quality.

Consequently, the most common approach to evaluate supervised classification algorithms today is to assess this single criterion (accuracy) using some selected metric. In many cases, the choice of metric is often based on the statistical or mathematical qualities (e.g.: AUC is chosen over success rate since the latter can be overly optimistic when the class distribution is skewed). At the same time, however, it is argued that the evaluation metric must represent something we care about [2]. One difficulty is then the diversity of the groups of people who must be considered in the decision on what to care about. Take the individual researcher, the research community of a certain discipline, and the end users of a certain piece of software as examples of groups of people that would probably care about quite different aspects of the solution to a problem. The conclusion must be that, it is unreasonable to expect that the concerns of all groups of people can be captured by one single metric. In fact, it is without doubt difficult to capture the different concerns by focusing on a single criterion no matter which criterion is used. Thus, for many applications, there is a need to identify and evaluate application-specific criteria to appropriately determine algorithm or classifier quality.

Moreover, some application-specific trade-offs can only be captured by using multiple criteria and perhaps even multiple evaluation metrics for each criterion. Last but not least, we stress the importance of defining bounds for acceptable metric results so that algorithms and classifiers are not just evaluated but can also be determined to be valid or invalid for a particular application. To summarize, we recognize the need for a systematic approach to validation and evaluation in which more care is taken to consider the context and requirements 
of the application at hand.

\section{A. Aim and Scope}

The aim of this paper is to present and demonstrate the use of a framework for systematic and application-oriented validation and evaluation of supervised learning algorithms and classifiers. The focus is on the description and demonstration of this framework. Thus, the unraveling of an exhaustive list of extensively described criteria and their corresponding metrics is outside the scope of the presented study. However, examples will be given of both possible criteria and related metrics.

\section{B. Outline}

The remainder of the paper is organized as follows: in Section II, we provide a more extensive analysis of the topic of evaluation criteria and review related work. Section III then presents the framework. In Section IV, we report on a case study in which the framework is applied to validate and evaluate a set of learning algorithms according to the requirements of a spyware detection tool. Lastly, in Section V, we draw conclusions and give some pointers to future work.

\section{BACKGROUND}

The no-free-lunch theorems [3] stipulate that all algorithms perform equally well averaged over all possible problems, i.e., no algorithm is superior for all problems. We argue that a relaxed analogy can be made to evaluation metrics in the sense that no single metric is superior for all problems, i.e., that all metrics are equally good taken over all possible problems. However, as mentioned earlier, it has been argued that some evaluation metrics are more appropriate than others, given a particular type of problem [1]. For example, a low false positives rate may be more important than a high success rate for a certain application.

Moreover, there are often space and time requirements in real-world applications that prohibit the use of many popular learning techniques, for example, when test sets are extremely large or where storage space or computational power is severely limited [4]. Thus, in order to select appropriate classifiers or algorithms for such applications we also need to evaluate performance in terms of space and time. We make a distinction between criteria and metrics in the sense that the latter are used to evaluate the former. Accuracy, space, and time could all be regarded as sub criteria of the performance criterion but it makes sense to discuss these sub-criteria explicitly as distinct criteria because the term performance is frequently used to denote accuracy.

If the goal is to discover new knowledge to be used in human decision making there might be completely different criteria to consider in addition to performance. For example, comprehensibility may relate to how well we understand the classifier and the decision process. In addition, a crucial aspect of data mining is that the discovered knowledge should be somehow interesting. This is related to the interestingness criterion [5], which has to do with the unexpectedness, usefulness and novelty of the discovered knowledge. Comprehensibility may also be associated with Complexity [6] in that an intricate or complex model is intuitively more difficult to comprehend than a simple model.

We have now highlighted some examples of criteria that can be important to evaluate but we believe there are many more to consider for various domains. As a consequence, we argue that evaluation criteria should be selected on the basis of the problem at hand. Moreover, it seems plausible that one should first decide on what criteria to evaluate before selecting metrics. This might sound obvious, but many studies seem to assume that accuracy is the only criterion worth evaluating.

Arguably, there is a need for; (i) common definitions of relevant evaluation criteria and (ii) systematic approaches to associate criteria with suitable metrics. The requirements of real-world applications often imply that a suitable trade-off between several important criteria is desired. For example, it has been noted that the accuracy and comprehensibility of rules are often in conflict with each other [5]. Thus, depending on the application, the evaluation may involve trading off multiple criteria. Since metrics are used to evaluate criteria, this means that a systematic approach is needed to trade off multiple metrics for these cases.

We believe that there is a fundamental problem with some of the proposed multi-criteria metrics in that they are usually based on a static set of metrics. As discussed in [7], one study proposes the SAR metric, which combines squared error, success rate, and AUC. This metric may be suitable for a certain application, however, it cannot be used to compare other criteria.

Thus, we need a standardized way to trade off multiple metrics but we would like the actual choice of which metrics to combine to be decided based on the particular application at hand. We therefore suggest the use of Generic Multi-Criteria (GMC) metrics, which dictate how to integrate metrics but do not specify what metrics to include. It could be argued that there is no need for GMC metrics and that the integration could instead be tailor-made for the application at hand. However, we argue that the benefits from using a GMC metric is that different instances of such a metric could be more easily compared and refined across studies and applications. In addition, the use of a GMC metric shifts the focus from integration issues to application issues.

The GMC metric approach also simplifies the development and use of what we refer to as metric-based learning algorithms, that is, algorithms that take a metric as input and try to learn a classifier with optimal performance with respect to this metric. A metric-based algorithm that is based on a GMC metric can thus be tailored for a particular application by selecting relevant metrics and specifying trade-offs.

We have discussed several aspects of application-oriented evaluation. and would like to stress the importance of understanding the characteristics, requirements, restrictions, goals, and the context of the application to be able to properly select suitable criteria and metrics and to define meaningful tradeoffs. Unfortunately, these aspects are covered in an ad-hoc manner any many studies if covered at all. 


\section{A. Related Work}

A number of studies address user-oriented and applicationoriented evaluation of classifiers and learning algorithms. One study presents a multi-criteria metric for learning algorithm evaluation [8] and define the efficiency of an algorithm as its weighted positive properties (for example, understandability) divided by its weighted negative properties (for example, computation time). The metric weights for the efficiency metric are decided by an optimization algorithm in order to calculate objective weights. The problem with this approach is that these objective weights might not contribute to a realistic representation of the studied application.

Moreover, the authors do not present any approaches to select metrics based on a particular application. In a followup paper an approach is suggested to measure qualitative properties, such as understandability, with the efficiency metric [9]. In addition, the paper introduces personalized algorithm evaluation, which is basically a collection of approaches to specify user preferences about restrictions and acceptable ranges on different metrics. The personalized evaluation approaches enable the user to influence the weights to reflect, for example, that understandability is more important than accuracy. However, one of the main problems is that the user preferences are defined as linear equalities of inputs and outputs, which is not very intuitive. The paper features some discussion on how to quantify qualitative metrics, however, no information is given about how to select relevant metrics. There exist, to our knowledge, no systematic approaches to evaluate learning algorithms according to multiple criteria and on the basis of a particular application.

\section{THE APPROVE FRAMEWORK}

Our framework is based on concepts from software engineering. Thus, it is beneficial to begin by introducing three related definitions. Firstly: given that we regard either a learning algorithm or a classifier as an item,

Definition 1. (A quality attribute is a) feature or characteristic that affects an items quality ${ }^{1}$.

In order for Definition 1 to be meaningful in the applicationoriented context, we define the meaning of quality as it pertains to an item:

Definition 2. (Quality is the) degree to which a component, system or process meets specified requirements and/or user/customer needs and expectations ${ }^{2}$.

Quality attributes are evaluated using quality metrics. For our purposes, the following definition of metrics is applicable:

Definition 3. (A metric is a) measurement scale and the method used for measurement ${ }^{3}$.

In summary, one or more metrics may be used to evaluate an algorithm (or classifier) according to a certain quality attribute,

\footnotetext{
${ }^{1}$ IEEE Standard Glossary of Software Engineering Terminology

${ }^{2}$ IEEE Standard Glossary of Software Engineering Terminology

${ }^{3}$ ISO/IEC 14598-1
}

i.e., an evaluation criterion. The scalar result of a metric is a quantification of the degree to which the algorithm or classifier meets the application requirements with regard to a certain quality. As discussed earlier, there might be a need to evaluate more than one quality attribute to obtain a complete assessment of the quality of an algorithm with respect to the studied application. The APPrOVE framework is really a process, which includes four sequential steps to perform: i) The evaluation problem is formalized by identifying the quality attributes that are relevant to the application at hand, ii) the identified quality attributes are prioritized according to their relative importance to the problem, iii) one or more suitable metrics are selected for evaluating each attribute, and iv) a multi-criteria validation and evaluation is performed of the candidate algorithms/classifiers using the selected metrics, taking into consideration the acceptable bounds for each metric.

Intuitively, the usefulness of the outcome of applying the framework depends on a fruitful collaboration between the designer/developer and the domain expert. In essence, we want to form an idea of the value of a learning algorithm or a classifier with respect to some problem (evaluation) and to check or prove the validity of the algorithm or classifier, both with respect to a certain application, Thus, our framework is called APPlication-Oriented Validation and Evaluation (APPrOVE). Let us review each step of APPrOVE in detail.

\section{A. Identification of Quality Attributes}

The process of identifying relevant quality attributes for the problem at hand should be simple. However, quality attributes can be defined on a number of abstraction levels. For example, depending on the characteristics of the problem, one may need to evaluate training-related (algorithmic) properties or testingrelated (classifier-specific) properties [10]. Moreover, some attributes can only be subjectively evaluated while others are associated with objective metrics. We suggest the use of a list of pairs of quality attributes and concise descriptions, as shown in Table I, and in which the quality attributes correspond directly to tangible qualities. It is then possible to browse the list to find the attributes that best match the (in)formal application requirements or objectives.

\section{B. Prioritization of Quality Attributes}

Suppose a real-world application of supervised learning is studied and several important quality attributes are identified. It is then improbable that these attributes are of equal importance. In order to take the difference in attribute importance into account in a meaningful manner, a systematic prioritization of the quality attributes is desirable.

There are quite a few methods available that perform systematic prioritization. The quantitative multi-criteria validation and evaluation method, which is the final step in the framework, makes use of individual metric weights. Since each metric is associated with a quality attribute, these weights can 
TABLE I

EXAMPLES OF QUALITY ATTRIBUTES

\begin{tabular}{ll}
\hline Attribute & The state or quality of being \\
\hline Accuracy & correct and precise \\
Availability & $\begin{array}{l}\text { operational when accessed } \\
\text { intricate or complicated }\end{array}$ \\
Complexity & able to be understood, intelligible \\
Consistency & uniform and free from contradiction \\
Efficiency & efficient (e.g., in terms of the ratio of useful \\
& work performed to the total resources ex- \\
& pended \\
Flexibility & easy to adjust to adapt to new situations \\
Interpretability & in appropriate language and units \\
Understandability & without ambiguity and easily comprehended \\
\hline
\end{tabular}

The definitions have been obtained from IEEE Standard Glossary of Software Engineering Terminology, the New Oxford American Dictionary, and from a study on data quality factors [11].

TABLE II

PAIRWISE COMPARISON ORDINAL SCALE

\begin{tabular}{ll}
\hline Value $^{1}$ & Meaning \\
\hline 1 & Attribute $k$ and $q$ are of equal importance \\
3 & Attribute $k$ is weakly more important than $q$ \\
5 & Attribute $k$ is strongly more important than $q$ \\
7 & Attribute $k$ is very strongly more important than $q$ \\
9 & Attribute $k$ is absolutely more important than $q$ \\
\hline \hline
\end{tabular}

A common configuration of the pairwise comparison ordinal scale used by the Analytic Hierarchy Process.

1 The numbers $2,4,6$, and 8 represent intermediate values.

also be regarded as quality attribute weights ${ }^{4}$. The Analytic Hierchary Process (AHP) [12] is therefore utilized in the presented study based on its compliance with these requirements as well as its successful use in similar prioritization tasks [13], [14].

1) The Analytic Hierarchy Process: AHP is executed by performing pairwise comparisons of a selected set of objectives, organized hierarchically into main objectives and sub objectives on as many levels as deemed suitable for the problem at hand. To limit the scope of the paper, we regard each identified quality attribute as a main objective.

For an arbitrary prioritization task with $n$ identified main attributes, the first step of AHP is to generate a $n \times n$ matrix, $O$, with pairwise comparison values. The attributes are now organized alphabetically and used as column and row labels for $O$. Let $k$ and $q$ denote index variables and let $\forall(k=$ q) $O_{k q}=1$. Using the ordinal scale provided in Table II, and for each attribute, $k$, that is considered to be at least of equal importance to another attribute, $q$, assign a suitable value, $v$, to $O_{k q}$. Correspondingly, let $O_{q k}=1 / v$.

The second step of AHP is to calculate the overall importance of each attribute. This step is performed by creating a

\footnotetext{
${ }^{4}$ In the simplest and perhaps most common case, one metric is selected for each identified quality attribute. However, the presented framework as such, is not limited to accomodating this case; in fact, both the framework and the applied prioritization method can handle multiple metrics for each quality attribute.
}

$n \times n$ attribute weight matrix, $P$. The value of each entry, $P_{k q}$, of this matrix is then calculated by dividing the corresponding pairwise comparison value, $O_{k q}$, with the sum of pairwise comparison values in the column the entry appears in. The overall importance, or weight, of each attribute is then obtained by calculating the average of the values in each row. Thus, the sum of attribute weights is 1 , which is a prerequisite for the employed multi-criteria evaluation method.

For the purpose of the presented study, the process described above is sufficient. However, it is possible to introduce sub attributes and even associated metrics. The described calculations are then repeated for each set of sub attributes to each attribute and each set of metrics associated with each sub attribute.

\section{Selection of Metrics}

As described in Section II, a certain quality attribute may be evaluated using various metrics, depending on the problem at hand. The question of how to select a suitable metric for a specific quality attribute is out of scope in this paper. There are many studies to refer to for readers interest in this topic (see, for example [1], [10]). For the purpose of the presented framework, it is sufficient to know that several aspects need to be taken into account when selecting metrics for a particular quality attribute. For example, some metrics are generic and may be used for all types of supervised algorithms (e.g., success rate, training time) while others are specific to a certain type of algorithm or classifier type (e.g., tree size, rule size). Additionally, some metrics may be more or less reliable depending on the type and distribution of data (e.g., success rate vs. AUC). Our multi-criteria evaluation method also expects each metric to have a particular range, which will be explained in the next sub section.

\section{Application-oriented Validation and Evaluation}

In previous work [7], we identified a number of attractive properties of existing multi-criteria evaluation metrics and presented a generic multi-criteria metric that was designed with these properties in mind. This metric, called the Candidate Evaluation Function (CEF), has the main purpose of combining an arbitrary number of individual metrics into a single quantity. In this study, we use a revised version [15] of CEF.

CEF normalizes the metrics in order to get a uniform output domain. It is also possible to specify explicit weights for each metric to ensure that application-specific trade-offs can be properly represented. CEF itself does not dictate which metrics to use; it merely dictates how metrics are combined. Finally, CEF makes it possible to specify the acceptable range for each metric, pertaining to a particular application, to allow for validation.

We define $m_{j}$ as a metric with index $j$ from an index set, $J$, over the selected set of metrics. Each metric is associated with a weight, $w_{j}$, and an acceptable range, $r=\left[b_{j}^{l}, b_{j}^{u}\right]$. The lower bound, $b_{j}^{l}$, denotes the least desired acceptable score. Similarly, the upper bound, $b_{j}^{u}$, denotes the desired score. Note 
that, in the original CEF definition a metric was normalized according to the best and worst score of that particular metric obtained from the studied set of classifiers. The revised version of CEF normalizes metrics using the lower and upper bound of the acceptable range to generate a distribution from 0 (least desired) to 1. CEF is now defined as specified in Equation 1.

$$
\operatorname{CEF}(c, D)=\left\{\begin{array}{l}
0: \exists j\left(\bar{m}_{j}(c, D)<0\right) \\
\sum_{j \in J} w_{j} \bar{m}_{j}(c, D) \text { otherwise }
\end{array}\right.
$$$$
\text { where } \sum_{j \in J} w_{j}=1 \text { and }
$$

$$
\bar{m}_{j}(c, D)=\left\{\begin{array}{l}
1: \frac{m_{j}-b_{j}^{l}}{b_{j}^{u}-b_{j}^{l}}>1 \\
\frac{m_{j}-b_{j}^{l}}{b_{j}^{u}-b_{j}^{l}} \text { otherwise }
\end{array} .\right.
$$

This concludes the presentation of the framework. We will now present a case study in which we apply the framework to a real-world problem.

\section{CASE Study}

The amount of spyware-associated software is ever increasing. To avoid legal repercussions, the vendors need to inform users about inclusion of spyware via the End User License Agreement (EULA) during the installation of a software product. However, this information is intentionally written in a way which is difficult to comprehend. In fact, it seems likely that most users take their chances by installing downloaded applications without reading the EULA at all.

In previous work [16], we have investigated the relationship between the contents of End User License Agreements (EULAs) and the legitimacy of the associated software applications. For this purpose, we collected a data set featuring 996 EULA instances of legitimate (good) and spywareassociated (bad) software. The experimental results revealed that a majority of the evaluated supervised learning algorithms significantly outperformed a baseline classifier, indicating the feasability of our approach. Since the approach seems to be successful in detecting the possible inclusion of spyware even before program installation, it could arguably be implemented as part of an end-user spyware prevention tool that could recommend users on whether to install or abort the installation of a certain product on the basis of the existence or absence of spyware components.

One important question, that need to be answered before developing the prevention tool, is which supervised learning algorithm to use in the EULA classification engine. In the aforementioned experiment, the studied algorithms were evaluated according to the accuracy quality attribute (using success rate and the AUC metric). However, in a real-world spyware detection scenario, there are most certainly other important qualities to consider as well. We now use APPrOVE to be able to draw conclusions about which algorithm to include in the prevention tool.

Informally (and hypothetically), we may desire the following: the prevention tool needs to be accurate in its classifications to allow for correct recommendations to the user, the

\section{Accuracy Complexity

\begin{tabular}{|c|c|c|}
\hline Accuracy & & Efficiency \\
\hline $\begin{array}{lll}9 & 7 & 5\end{array}$ & 31 & $\begin{array}{llll}3 & 5 & 7 & 9\end{array}$ \\
\hline fficiency & & Complexity \\
\hline $9 \pi$ & 31 & 357 \\
\hline
\end{tabular} \\ $\begin{array}{lllllllll}9 & 7 & 5 & 3 & 1 & 3 & 5 & 7 & 9\end{array}$}

Fig. 1. Domain expert assessment of the relative importance for each pair of quality attributes

decision process of the tool should be easily comprehended by users to empower their ability to make an informed choice about whether to install a particular piece of software, and the tool needs to be efficient in that it must be able to generate classifiers quickly from large amounts of data. One of the intended features of the tool is that it should visualize the classifier to make the decision process transparent to the user. A choice is made to focus on decision tree inducers in order to implement this feature.

We identify: complexity, accuracy, and efficiency as relevant attributes for determining the quality of a candidate algorithm with respect to the requirements posed on the prevention tool. Equation 2 shows the results of the first step of AHP, which involves getting domain experts to assess the relative importance of each quality attribute in comparison to the other attributes and then generating a pairwise comparison value matrix (left) and then an attribute weight matrix (right). In Figure 1, the assessment of the domain experts is shown. There is in fact nine possible pairs of quality attributes. However, three of the pairs correspond to comparisons of identical attributes and three pairs correspond to the pairs in Figure 1 but with the order reversed. These six pairs are obviously of no importance. As can be seen in the third paired comparison, an intermediate value (6) is selected.

$$
\left[\begin{array}{ccc}
1 & 1 / 9 & 1 / 6 \\
9 & 1 & 5 \\
6 & 1 / 5 & 1
\end{array}\right] \rightarrow\left[\begin{array}{ccc}
0.063 & 0.085 & 0.027 \\
0.563 & 0.763 & 0.811 \\
0.375 & 0.153 & 0.162
\end{array}\right]
$$

By calculating the average for each row in the right matrix of Equation 2, the weights $0.058,0.712$, and 0.230 are obtained for Complexity, Accuracy, and Efficiency, respectively. Domain experts then select one metric for each identified attribute: tree size (complexity), success rate (accuracy), and training time (efficiency). Whereas success rate and training time are generic metrics, the tree size metric is specific to decision tree algorithms. The results from the experiment are presented in Table III. Recall that, these results represent raw test scores. In order to perform CEF evaluation, each score is normalized subject to the defined boundaries. If a metric score 
TABLE III

EXPERIMENTAL RESULTS

\begin{tabular}{lrrr}
\hline Learning algorithm & Tree size & Success rate & Training time \\
\hline J48 & 15 & 0.971 & 8.61 \\
RandomTree & 173 & 0.945 & 3.37 \\
REPTree & 5 & 0.970 & 2.37 \\
ADTree & 31 & 0.977 & 69.26 \\
\hline
\end{tabular}

Test results for the metrics associated with Complexity, Accuracy, and Efficiency.

TABLE IV

MULTI-CRITERIA EVALUATION RESULTS

\begin{tabular}{lrrrr}
\hline Algorithm & Complexity & Accuracy & Efficiency & CEF \\
\hline J48 & 0.029 & 0.691 & 0.213 & 0.934 \\
RandomTree & 0.000 & 0.673 & 0.223 & 0.000 \\
REPTree & 0.048 & 0.691 & 0.225 & 0.964 \\
ADTree & 0.000 & 0.696 & 0.097 & 0.000 \\
\hline
\end{tabular}

Normalized metric scores for each pair of quality attribute and algorithm as well as CEF evaluation scores for each algorithm.

is outside of the acceptable range, the score is set to zero, which ultimately results in a CEF evaluation score of zero, which means that the corresponding algorithm is not valid for the studied application. The normalized metric scores and the CEF evaluation score for each algorithm can be viewed in Table IV. RandomTree and ADTree fail in meeting the minimum complexity requirements, which leads to zeroed out CEF scores. We therefore conclude that these algorithms are not valid choices for the studied problem. J48 and REPTree are both valid, but to different degrees of quality. There is no difference in normalized success rate. However, REPTree outscores J48 in terms of having a simpler classifier model and a lower training time. Thus, the two algorithms are equally accurate but REPTree is less complex and more efficient. The total CEF score is also affected by the relative importance of each quality attribute in that the metric weights correspond to this importance. We decide to select REPTree for inclusion in the EULA classification engine of the spyware prevention tool.

\section{CONCLusions And Future Work}

Machine learning algorithms are increasingly used in realworld applications. For classification problems, the most common success criterion is accuracy, which may be evaluated using the area under the ROC curve, success rate, or some other metric. However, the use of accuracy as the only criterion does not capture the requirements of very many real-world applications. The question is, how to identify, prioritize, and evaluate multiple (perhaps conflicting) criteria. There is arguably a need for a systematic and application-oriented evaluation approach. This paper presents a framework, called APPrOVE, which involves four sequential steps: i) identification of quality attributes, ii) attribute prioritization, iii) metric selection, and iv) validation and evaluation. A case study is conducted to demonstrate the use of APPrOVE to validate and evaluate a set of candidate algorithms for a particular application.
There are several interesting directions for future work: in APPrOVE, we prioritize the quality attributes before selecting evaluation metrics. However, it is quite possible to reverse the order of these steps. One benefit of this change in procedure is that the analytic hierarchy process can be extended to prioritize the metrics associated with each quality attribute as well. APPrOVE also involves the use of a multi-criteria metric, which yields a single quantity result. By enforcing the use of a univariate multi-criteria metric, rather than a multivariate multi-criteria method, it is possible to replace the learning metric of some of the existing supervised learning algorithms to optimize their performance toward application requirements during classifier generation. In order to investigate this approach, we are currently working on re-designing a set of selected learning algorithms to allow for replacement of their learning metrics.

\section{REFERENCES}

[1] R. Caruana and A. Niculescu-Mizil, "An empirical comparison of supervised learning algorithms," in 23rd International Conference on Machine Learning. New York City, NY, USA: ACM Press, 2006, pp. 161-168.

[2] C. Drummond, "Machine learning as an experimental science (revisited)," in AAAI Workshop on Evaluation Methods for Machine Learning. Menlo Park, CA, USA: AAAI Press, 2006, pp. 1-5.

[3] D. H. Wolpert, "Off-training-set error and a priori distinctions between learning algorithms," Sante Fe Institute, Santa Fe, NM, USA, Tech. Rep. SFI-TR 95-01-003, 1995.

[4] C. Bucila, R. Caruana, and A. Niculescu-Mizil, "Model compression," in 12th ACM SIGKDD International Conference on Knowledge Discovery and Data Mining. ACM Press, 2006.

[5] A. Freitas, "On objective measures of rule interestingness," in Second European Symposium on Principles of Data Mining \& Knowledge Discovery. Springer, 1998.

[6] B. Gaines, "Transforming rules and trees into comprehensible knowledge structures," in Advances in Knowledge Discovery and Data Mining, U. F. et al., Ed. Cambridge, Mass.: MIT Press, 1996, pp. 205-226.

[7] N. Lavesson and P. Davidsson, "Generic methods for multi-criteria evaluation," in Eighth SIAM International Conference on Data Mining. SIAM Press, 2008.

[8] G. Nakhaeizadeh and A. Schnabl, "Development of multi-criteria metrics for evaluation of data mining algorithms," in Third international conference on knowledge discovery and data mining. AAAI Press, 1997, pp. 37-42.

[9] - "Towards the personalization of algorithms evaluation in data mining," in Fourth international conference on knowledge discovery and data mining. AAAI Press, 1998, pp. 289-293.

[10] N. Lavesson and P. Davidsson, "Evaluating learning algorithms and classifiers," Intelligent Information \& Database Systems, vol. 1, no. 1, pp. 37-52, 2007.

[11] R. W. Wang and D. M. Strong, "Beyond accuracy: What data quality means to data consumers," Journal of Management Information Systems, vol. 12, no. 4, 1996.

[12] T. L. Saaty, The Analytic Hierarchy Process: Planning, Priority Setting, Resource Allocation. McGraw-Hill, 1980.

[13] O. S. Vaidya and S. Kumar, "Analytic hierarchy process: An overview of applications," European Journal of Operational Research, vol. 169, no. 1, pp. 1-29, 2004.

[14] C. Wohlin and A. A. Andrews, "Prioritizing and assessing software project success factors and project characteristics using subjective data," Empirical Software Engineering, vol. 8, no. 3, pp. 285-308, 2003.

[15] N. Lavesson and P. Davidsson, "AMORI: A metric-based one rule inducer," in Ninth SIAM International Conference on Data Mining. SIAM Press, 2009.

[16] N. Lavesson, M. Boldt, P. Davidsson, and A. Jacobsson, "Learning to detect spyware using end user license agreements," Knowledge and Information Systems, 2010. 